and scored urine proteinuria, skin lesions and lymphadenopathy. At the end of the treatment interval, kidney, lung, spleen, skin, plasma and urine samples were collected and shipped to the coordinating study center for blinded analysis. All histopathological assessments were performed by an independent kidney pathology institute (RWTH Aachen, Germany) in a blinded fashion. The pre-set primary endpoint was urinary protein/creatinine ratio. Secondary endpoint analyses included plasma auto-antibodies levels, kidney histologic scores (activity and chronicity indices). Glomerular filtration rate and flow cytometry of splenocytes was performed on subgroups of mice at single centers.

Results A total of 56 mice were used, of which 13 in Madrid, 15 in Barcelona, 14 in Freiburg, and 14 in Munich. Mice were randomly assigned to baricitinib treatment $(n=28)$ or vehicle $(n=28)$. At treatment initiation, the average score of proteinuria (tested by sticks 0-4), skin lesion (0-4), and lymphadenopathy (0-6) for baricitinib group were $1.38 \pm 0.99$, $0.95 \pm 1.28$, and $2.10 \pm 1.80$, respectively; above scores for vehicle group were $1.45 \pm 1.10,0.77 \pm 1.02$, and $1.81 \pm$ 2.02 , respectively. Data analysis is ongoing and will be presented at the conference.

Conclusions Preclinical double-blind, randomized, controlled, multicenter trials are a novel tool in preclinical drug testing that might help to predict the outcome of randomized clinical trials.

\section{P135 A PHASE I, FIRST-IN-HUMAN STUDY TO ASSESS THE SAFETY, PHARMACOKINETICS AND PHARMACODYNAMICS OF SINGLE AND MULTIPLE ASCENDING DOSES OF M5049, A DUAL ANTAGONIST OF TLR7/8, IN HEALTHY SUBJECTS}

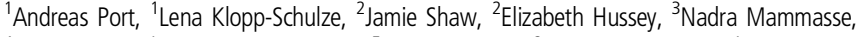
${ }^{4}$ Ying Zhang, ${ }^{1}$ Angelika Bachmann, ${ }^{5}$ Christian Reh, ${ }^{2}$ Kosalaram Goteti. ${ }^{1}$ Translational Medicine, Merck KGaA, Darmstadt, Germany; ${ }^{2}$ Translational Medicine; ${ }^{3}$ Biostatistics, Cytel, Paris, France; ${ }^{4}$ Global Patient Safety, EMD Serono Research and Development Institute*, Billerica, USA; ${ }^{5}$ Nuvisan GmbH, Neu-Ulm, Germany; *A business of Merck KGA, Darmstadt, Germany
\end{abstract}

\subsection{6/lupus-2020-eurolupus. 179}

Background Toll-like receptors 7 and 8 (TLR7/8) are widely expressed in a variety of immune cells and detect singlestranded RNAs, activating signalling cascades which trigger an immune response. Aberrant TLR7/8 activation by RNA-containing autoantibodies can result in autoimmunity. M5049 is an oral small molecule TLR7/8 antagonist which inhibits TLR7/8 activity in vitro and ex vivo and has demonstrated efficacy in mouse SLE models, suggesting potential to inhibit pathological immune complex activities in SLE patients.

Methods This was a phase I, randomized, double-blind, placebo-controlled (3:1), single and multiple ascending dose (SAD and MAD) study of oral M5049 conducted in healthy participants. SAD cohorts received a single dose of M5049 (1, 3, 9, 25, 50, 100 and $200 \mathrm{mg}$ ) or placebo, and MAD cohorts received M5049 (9, 25, $200 \mathrm{mg}$ once daily, 25 and $50 \mathrm{mg}$ twice daily) or placebo for 14 days. A sentinel dosing strategy was used in SAD cohorts. The study assessed safety, tolerability, pharmacokinetics (PK), and pharmacodynamics (ex vivostimulated cytokine secretion). Food effect was assessed in the 25 mg SAD cohort.

Results Preliminary results showed that M5049 was well-tolerated over the dosing interval, with no significant or doselimiting adverse events observed to date. PK parameters were linear and dose-proportional from 1 to $200 \mathrm{mg}$, with higher clearance and shorter half-life than predicted based on preclinical studies. Exposure-dependent inhibition of $e x$ vivo-stimulated IL-6 secretion was observed, with maximum inhibition achieved at $200 \mathrm{mg}$. PK results indicate a slight food effect.

Conclusions M5049 was well-tolerated with no safety signals in healthy participants, warranting further investigation as a potential treatment for autoimmune diseases, such as SLE.

\section{P136 SLEDAI RESPONSE PREDICTION TO BELIMUMAB THERAPY BY BASELINE LEVELS OF BLYS, APRIL AND CD8+ EFFECTOR MEMORY T-CELLS}

${ }^{1}$ Francesca Regola, ' Silvia Piantoni, ${ }^{1}$ Laura Andreoli, ${ }^{2}$ Torsten Lowin, ${ }^{1}$ Paolo Airò, ${ }^{1}$ Franco Franceschini, ${ }^{1}$ Angela Tincani, ${ }^{2}$ Georg Pongratz. ${ }^{1}$ Rheumatology and Clinical Immunology, ASST Spedali Civili, University of Brescia, Brescia, Italy; ${ }^{2}$ Rheumatology and Hiller Research Center for Rheumatology, University Hospital Düsseldorf, Düsseldorf, Germany

\subsection{6/lupus-2020-eurolupus. 180}

Background Systemic Lupus Erythematosus (SLE) patients show high levels of BLyS (B-lymphocyte stimulator) and other cytokines belonging to the tumor necrosis factor (TNF) superfamily ${ }^{1}$. Belimumab, a monoclonal antibody against BLyS, mainly targets B-cells ${ }^{2}$, but a BLyS-dependent T-cell activation pathway has been demonstrated ${ }^{3}$. Clinical studies showed that anti-DNA antibodies and complement levels at baseline are predictors of response to Belimumab. Our study aims at identifying other biomarkers as response predictors.

Methods Twenty-one SLE patients received Belimumab. Biomarkers belonging to the TNF superfamily (BLyS, APRIL, sBCMA, sCD40L, sTACI, TWEAK) were tested by ELISA in all patients and lymphocyte immunophenotyping was performed by flow cytometry in ten subjects at baseline and every six months. SLE-disease activity was assessed by SLEDAI-2K score.

Results BLyS and APRIL baseline serum levels and the number of CD3+CD8 + effector memory T-cells were correlated positively with SLEDAI-2K improvement after 12 months of treatment (Pearson correlation $=0.535 \quad(p=0.015), \quad 0.504$ $(\mathrm{p}=0.023)$ and $0.654(\mathrm{p}=0.040))$. After backwards exclusion from linear regression analysis including SLEDAI-2K, effector T-cell relative number and BLyS or APRIL at baseline, only APRIL remained as significant independent predictor of SLEDAI-2K improvement after 12 months (adjusted R square $=0.649 ; \mathrm{p}=0.025)$. After comparing TNF-family members levels and SLEDAI-2K at baseline, only BLyS showed the best predictive value (adjusted $\mathrm{R} 0.564$, $\mathrm{p}<0.001)$.

Conclusions In our cohort of SLE patients, baseline level of APRIL together with percentage of CD3+CD8+ effector memory T-cells, or BLyS serum level alone resulted as best predictors of response to Belimumab. Considering that immunophenotyping is rarely done in clinical practice, BLyS baseline serum levels alone could be used routinely as a good predictor of response, as suggested by post-hoc analyses of the BLISS study.

\section{REFERENCES}

1. $\mathrm{Ng} \mathrm{LG}$, et al. J Immunol, 2004.

2. Ramsköld D, et al. EBioMedicine, 2019.

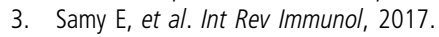

